celui du virus polyome, agit comme un activateur sur la séquence stimulatrice $d u$ gène d'immunoglobuline transfectée dans des fibroblastes de souris, probablement en levant l'inhibition par les facteurs de régulation négative (figure I). Une même protéine peut donc être inhibitrice pour certains enhancers et activatrice pour d'autres. Un tel modèle, s'il était généralisé, permettrait de simplifier un peu les mécanismes impliqués dans la régulation des gènes spécifiques d'un tissu différencié : les facteurs "activateurs" des séquences stimulatrices de gènes exprimés dans ces tissus pourraient être aussi les "inhibiteurs " des enhancers d'autres gènes dont l'expression n'est pas spécifique du tissu.

L'équipe de W. F. Rutter vient d'obtenir, sur un autre modèle, des résultats très proches de ceux d'E. Borelli. Un gène dont l'expression est contrôlée par les séquences régulatrices $d u$ gène de l'insuline ne s'exprime pas dans des cellules fibroblastiques de singe, sauf si ces cellules contiennent en même temps un large excès $d u$ enhancer $d u$ gène de l'insuline, qui est supposé épuiser les facteurs inhibiteurs selon le schéma de la figure I [4].

Enfin, deux autres équipes ont rapporté que les séquences stimulatrices pouvaient être inhibées par un autre mécanisme que la fixation à leur niveau de facteurs diffusibles inhibiteurs. Il existe en amont des gènes c-myc [5] et de l'insuline de rat [6] des "silencers" (voir médecine) sciences $n^{\circ} 6$, vol. I, p. 335) inhibant en cis (c'est-à-dire lorsqu'ils sont localisés sur le même double brin d'ADN) l'activité stimulatrice de enhancers. Le rôle de ces séquences pourrait être, notamment, d'isoler les gènes de l'influence de séquences stimulatrices appartenant à des gènes adjacents.

Les mécanismes résumés ici pourront sembler bien complexes au lecteur, et, de fait, ils le sont. Ils constituent cependant, touche après touche, l'ébauche d'une des principales avancées scientifiques de notre siècle : le matériel génétique, ce qu'il est et la manière dont il fonctionne.

A. $\mathbf{K}$.

I. Borelli E, Hen R, Chambon P. Adenovirus-2 $\mathrm{E}_{1}$ A products repress enhancer-induced stimulation of transcription. Nature, 1984; 31 2 : 608-1 2. 2. Hen R, Borelli E, Fromental C, Sassone Corsi P, Chambon P. A mutated polyoma virus enhancer which is active in undifferentiated embryoma carcinoma cells is not repressed by adenovirus-2 $E_{1}$ A products. Nature $1986 ; 321$ : 249-51.

3. Borelli E, Hen R, Wasylyk C, Wasylyk B, Chambon $P$. The immunoglobulin heavy chain enhancer is stimulated by the adenovirus type-2 $\mathrm{E}_{1}$ A products in mouse fibroblasts. Proc Natl. Acad Sci USA 1986; $83: 2846-9$.

4. Nir U, Walker MD, Rutter, WJ. Regulation of rat insulin I gene expression: Evidence for negative regulation in nonpancreatic cells. Proc Natl Acad Sci USA 1986; 83: 3180-4.

5. Remmers EF, Yang JQ Marcu KB. A negative transcriptional control element located upstream of the murine $c$-myc gene. EMBO J I 986; 5 : 899-904.

6. Laimins L, Holmgren $M$, Khoury G. Transcriptional silencer element in rat repetitive sequences associated with the rat insulin I gene locus. Proc Natl Acad Sci USA, 1986; 83: 315I-5.

\title{
Benzodiazépines et rythmes
}

Les rythmes circadiens ont acquis une importance qui n'est plus à démontrer. Ils sont notamment sous l'influence de la lumière, mais les changements d'horaire ou de durée des périodes de lumière ne sont compensés que progressivement, d'où, par exemple, les difficultés qu'éprouvent de nombreuses personnes lors d'un décalage horaire. Des troubles du cycle veille-sommeil sont également très fréquents; ils peuvent être améliorés mais aussi parfois aggravés par des médicaments. Le poste de commande central se trouve probablement dans les noyaux suprachiasmatiques, et met en jeu des neurones contenant du GABA.

Parmi les médicaments capables de modifier les rythmes circadiens, ce sont les benzodiazépines, largement mises à contribution par médecins et malades, qui sont en première ligne. Par des expériences simples, Turek et Losee-Olson [I] d'Evanston (Illinois) ont montré l'effet du triazolam, une benzodiazépine utili- sée dans le traitement de l'insomnie, sur l'activité locomotrice chez le hamster doré. Les animaux sont placés dans des conditions de lumière définie (obscurité ou lumière prolongée). Ils reçoivent 2,5 $\mathrm{mg}$ de triazolam (dose en fait nettement supérieure à celles qu'on emploie chez l'homme) à huit moments de la journée également espacés. Cet agent actif provoque immédiatement un décalage dans les phases d'activité motrice d'environ une heure. Ce décalage peut consister en une avance ou un retard, selon l'horaire et les conditions de base (lumière ou obscurité). Sa durée dépend de façon précise de l'horaire des injections.

De tels travaux vont probablement revêtir une importance considérable dans les années à venir. Une convergence devrait en effet s'établir entre biologie moléculaire et neurophysiologie. Médecine/sciences a relaté $\left(n^{\circ} 4\right.$, vol. $\left.2, p .223\right)$ l'isolement d'un gène gouvernant certains rythmes biologiques chez la drosophile, et l'on est en droit de s'attendre, comme dans le cas des homéoboxes, à l'extension de telles découvertes aux animaux supérieurs. Sur un plan plus pratique, on devrait pouvoir en tirer des déductions thérapeutiques. L'usage de médicaments dotés de propriétés qui changent les rythmes circadiens est en effet une médaille avec son revers : il devrait être possible [2] de mettre au point des médicaments capables de décaler de manière précise le rythme veille-sommeil, permettant ainsi de supprimer par exemple les conséquences du décalage horaire. Mais leur connaissance inciterait également à la prudence dans le choix de médicaments psychotropes, susceptibles d'aggraver à terme des symptômes, comme l'insomnie, qu'ils sont supposés combattre.

J.-C. D.

I. Turek FW, Losee-Olson S.A benzodiazepine used in the treatment of insomnia phase-shifts the mammalian circadian clock. Nature I986; 321 : 167-8.

2. Winf ree AT. Benzodiazepines set the clock. Nature 1986; 32I : I I 4-5. 